Highlights

- Social wasps are considered as effective biocontrol agents

- Predation by a Polistinae was studied using a light trap to attract insect prey

- Foragers can carry $30.7 \%$ of their load capturing up to six small prey

- Large prey are cut into two pieces; the wasps return to gather the second piece

- Social facilitation, a primitive form of nestmate recruitment was noted 


\section{The predatory behavior of the Neotropical social wasp Polybia rejecta}

Alain Dejean ${ }^{\text {a, b, }{ }^{*}, \text { Héctor Rodríguez-Pérez }}{ }^{\mathrm{c}}$, James M. Carpenter ${ }^{\mathrm{d}}$, Frédéric Azémar ${ }^{\mathrm{a}}$, Bruno Corbara $^{\mathrm{e}}$

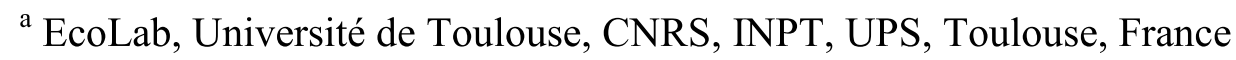

${ }^{\mathrm{b}}$ CNRS, UMR EcoFoG, AgroParisTech, Cirad, INRA, Université des Antilles, Université de Guyane, 97310 Kourou, France.

${ }^{\mathrm{c}}$ UMR CNRS 6553 ECOBIO, University of Rennes 1, 35042 Rennes Cedex, France.

${ }^{\mathrm{d}}$ Division of Invertebrate Zoology, American Museum of Natural History, Central Park West at 79th Street, New York, NY, 10024, USA

${ }^{\text {e }}$ Université Clermont Auvergne, CNRS, LMGE, F-63000 Clermont-Ferrand, France

* Corresponding author: Alain Dejean; Ecolab, Université Toulouse3, 118 Route de Narbonne, 31062 Toulouse, France.

Tel.: (33) 5615589 17; Fax: (33) 5615589 01. E-mail: alain.dejean@wanadoo.fr

Total number of words (title, text, acknowledgements, references and figure legends): 7176 


\section{ABSTRACT}

We experimentally studied the predatory behavior of Polybia rejecta (Vespidae, Polistinae, Epiponini) towards 2-88 mm-long insects attracted to a UV light trap. Foragers, which began to hunt at 6:30, selected 4-14 mm-long prey insects. Prey detection by sight by hovering wasps was confirmed using decoys. After the wasps landed and walked along a sinuous path, prey were detected by contact or from a distance $(1-3 \mathrm{~cm})$. This was followed by seizure, stinging (contrarily to most other known cases), prey manipulation and retrieval. Prey that flew off might be caught in flight. The prey load, representing $30.7 \%$ of a forager's weight, was optimized by capturing up to six small prey or two medium-sized prey successively (both of which might be consumed in situ). The foragers cut off the wings of larger prey or cut them into two pieces and returned to gather the second piece. The handling time increased exponentially with the weight of the prey. Partial loading (i.e., retrieving a load much inferior to the maximum possible) was likely related to social facilitation, a form of nest-based recruitment that was demonstrated through the experimental elimination of local enhancement by removing foragers (both mechanisms favor the exploitation of favorable patches).

199 words $<200$

\section{Keywords}

Epiponine wasps, Polistinae, predation, prey selection, recruitment, Vespidae 


\section{Introduction}

Foraging is the basis for the survival, development and reproduction of individual animals. It permits their populations to endure while impacting other species, as seen through pollination, seed dispersal, parasitism and, for predators, the regulation of prey populations (Schoener, 1987; Kramer, 2001). To reduce the costs of food acquisition, animals must adapt their foraging behavior to spatially and temporally dynamic environments while avoiding their predators, so that theoretical models have been developed where the premise is that an optimization process results from natural selection (i.e., the notion of optimal foraging) (MacArthur and Pianka, 1966; Pyke et al., 1977).

In the case of a solitary predator a foraging bout includes the elements (1) search, (2) detection, (3) assessment resulting in pursuing or abandoning prey, (4) pursuit leading to prey contact and attack, (5) handling that includes mastering the prey, its preparation and consumption, and (6) processing or digestion (Kramer, 2001). Animals maximize benefits and minimize costs through an optimal diet based partly on the size of prey (i.e., a function of the time necessary to master them, or "handling time"), the choice of optimal patches to forage, the allocation of time spent in these patches, and the patterns of movement (i.e., foragers increase the sinuosity of their path and decrease their speed in favorable patches, known as "intensive search"; a patch is abandoned when the density of prey decreases). Therefore, when foraging, predators need to make decisions based on perceived cues related to prey density, quality and defenses, resulting in choices where risks are perceived as well as the possibility for inter- and intraspecific kleptobiosis (notion of decision rules; Iwaza et al., 1981; Stephens and Krebs, 1986; Kramer, 2001; Darracq et al., 2016).

Central place foragers, such as birds during the breeding season that return to their nests to feed their offspring or eusocial insects that feed their kin (i.e., nestmates and the brood of 
the colony), need to use navigation and memory to return to the nest and then to favorable patches (Orians and Pearson, 1979; Schoener, 1979; Hölldobler and Wilson, 1994; Sutton, 2015). In eusocial insects, the foraging bouts of solitary hunters are composed of (1) an outward trip, (2) search, (3) mastering the prey, (4) loading, (5) a homeward trip and (6) unloading (Kramer 2001). Although it can occur, the consumption of the prey is no longer the main objective of the forager. "Social foragers" have, in addition, the ability to inform their nestmates of the location of favorable patches (e.g., recruitment trails in ants and the waggle dance in honeybees) (Hölldobler and Wilson, 1994; Beekman and Dussutour, 2009). Loading can be optimized by retrieving large prey or successively capturing several small prey and retrieving them all at one time. Alternatively, partial loading often occurs when recruiting nestmates is needed to rapidly deplete a food source. Moreover, the theory predicts that when the load affects the duration of the homeward trip, the optimal load size decreases with an increasing distance to the nest (Schoener, 1979; Kasuya, 1982; Wetterer, 1989; Dornhauss et al., 2006).

Because most social wasps (e.g., Vespinae and Polistinae including the swarm-founding Epiponini) are generalist predators preying mostly on caterpillars and adult Diptera and so can be used as biological control agents (Raveret Richter, 2000; Donovan, 2003; Jeanne and Taylor, 2009; Bichara Filho et al., 2010; Santana et al., 2012) they constitute a good model for studying their predatory behavior. The foraging bouts of social wasps begin with a flight towards a potential prey habitat. Then, search flights are composed of highly irregular loops alternating with phases of inspection where individuals hover while oriented toward a visible resource (Raveret Richter and Jeanne, 1991; Collett, 1995; Jander, 1997; Stürzl et al., 2016). Although prey, particularly live, fast-moving insects, are mostly detected by sight, odors also play a role (Raveret Richter and Jeanne, 1985; Hendrich et al., 1994; Raw, 1998; Jeanne and Taylor, 2009; Couto et al., 2014). The prey capture behavior of Polistes dominula foragers is 
composed of the following phases: approach (generally walking), antennation, attack and biting various parts of the prey, butchering (slicing away pieces of flesh) and balling up (preparation of a small chunk of flesh to be transported). Finally, the ball of flesh is taken to the nest (Brown et al., 2012). The foragers of some Epiponini do not malaxate their prey, but rather transport them virtually intact or cut off only the non-edible parts, such as the wings; others retrieve only the most profitable part (e.g., the thorax of adult insects with the wing muscles), and still others cut large prey into pieces which are retrieved during successive flights (Jeanne, 1972; Hunt et al., 1987; Raveret Richter, 2000; Raveret Richter and Jeanne, 1991; Gomes et al., 2007; Jeanne and Taylor, 2009; Dejean et al., 2010, 2012; Polidori et al., 2013). Although foragers generally kill live prey by biting them, stinging, a facultative phase, is triggered when the prey struggle. This permits the wasps to economize their venom which is only used to master large and/or vigorous prey (Edwards, 1980; Olson, 2000).

Navigation using sunlight angles, visual landmarks in the environment and a cognitive map (Collett et al., 2016; Stürzl et al., 2016) permits the wasps to take a direct homeward path. Learning flights (i.e., the individuals back away from the goal in a series of arcs) occur just after leaving the nest and before leaving a large food resource, permitting the foragers to find them later (Zeil et al., 1996; Jander, 1997; Stürzl et al., 2016). Capable of 24-hour recall, foragers can undertake a series of return trips (Moreyra et al., 2016).

When moving to a new nest site, the Epiponini recruit nestmates by laying scent trails (Jeanne, 1981, 1991; Francescato et al., 1993; Howard et al., 2002), but this mechanism has never been observed during food recruitment (see Jeanne and Taylor, 2009). Yet, in Polybia occidentalis, naïve individuals in the nest use food scent cues from the carbohydrates brought in by successful foragers to orient themselves to food resources (social facilitation; Jeanne and Taylor 2009; Shettleworth, 2010). Furthermore, in the field, the presence of conspecifics at a site attracts the foragers of different polistine species (local enhancement or increased 
likelihood of visiting a place by virtue of observing others doing it) (Raveret Richter, 1990; Hrncir et al., 2007; Schueller et al., 2010; Shettleworth, 2010; Taylor et al., 2012; Schueller and Jeanne, 2012; for Vespinae, see Santoro et al., 2015). Also, group hunting, likely related to a form of recruitment, has been noted in the Epiponini, illustrating the possibility of cooperation and coordination (O’Donnell and Hunt, 2013). Yet, except for tandem flights possibly performed by Vespula germanica foragers (Lozada et al., 2016), these types of information signals do not direct recruited individuals from the nest to the specific location of a food resource.

We studied the predatory behavior of Polybia rejecta (Epiponini) aiming to determine (1) when the wasps forage, (2) if visual cues are used to detect prey by using decoys, (3) the size/weight range of selected prey, (4) the differences in capture behavior according to prey size, (5) the impact of the prey-predator weight ratio on handling time, and (6) experimental manipulations to test for social enhancement, a form of recruitment to food resources.

\section{Materials and Methods}

\subsection{Study sites and experimental set up}

This study was conducted at the Hydreco field station at Petit Saut, Sinnamary, French Guiana $\left(5^{\circ} 03^{\prime} 39^{\prime \prime} \mathrm{N} ; 5^{\circ} 02^{\prime} 36^{\prime \prime} \mathrm{W}\right)$ between 2009 (preliminary observations) and 2015. The climate is tropical moist, with $3500 \mathrm{~mm}$ of annual precipitation distributed over 280 days. There is a major drop in rainfall between July and November (dry season) and another shorter and more irregular dry period in March.

The study area, the outer western wall of the Hydreco laboratory, is situated $\approx 200 \mathrm{~m}$ downstream from the center of the Petit Saut dam and, at $\approx 30 \mathrm{~m}$ height, overlooks the right bank of the Sinnamary River. There, we set up one or the other of two light traps to attract 
nocturnal flying insects. The traps consisted of the white western wall $(4 \times 2 \mathrm{~m})$ or a white plastic sheet $(3 \times 2 \mathrm{~m})$ mounted on a frame, both lit by a self-ballasted 80 -watt mercury vapor lamp emitting both white and UV light. The wall or the white sheet were hidden from the seven wasp nests used in this study by the buildings of the research station; the distance separating these nests and the experimental set-up varied from $60.5 \mathrm{~m}$ to $105 \mathrm{~m}$.

Each series of observations and experiments was conducted during five consecutive nights without moonlight (two nights before and two nights after the new moon). The light traps attract numerous insects with small moths and small dipterans being particularly abundant. Because most of the insects attracted stay on the wall or the white sheet during the next day, we had the opportunity to work on the foraging behavior of $P$. rejecta wasps which began to hunt on the wall or the white sheet from the first morning of each experimental period (see Raveret Richter, 1990 for training social wasps to prey on furnished insects).

\subsection{The focal social wasp species}

These studies were conducted on the swarm-founding Neotropical social wasp Polybia rejecta (Fabricius, 1798) (Vespidae; Polistinae; Epiponini) (Fig. 1) whose nests, which are protected by an envelope, can reach $30 \mathrm{~cm}$ in diameter and $2 \mathrm{~m}$ in height, although most of them are less than $70 \mathrm{~cm}$ in height. These nests have frequently been noted as being associated with the large carton nests of the territorially dominant arboreal dolichoderine ant species Azteca chartifex. In this mutualistic association, the ants protect the wasps from army ant attacks, while the wasps protect the ant nests from bird attacks (Herre et al., 1984; Corbara et al., 2009; Somavilla et al., 2013; Le Guen et al., 2015).

Polybia rejecta individuals mainly prey on soft bodied arthropods, especially lepidopteran larvae, and are very aggressive toward humans, who are attacked from more than $15 \mathrm{~m}$ away without any "provocation"; their sting is very painful (Starr, 1985; Somavilla et al., 2013; Le 
Guen et al., 2015). Note that foraging workers are old individuals whose remaining lifespan is probably short: only 20-25 days in another Epiponini wasp species with large nests (O’Donnell and Jeanne, 1992).

\subsection{Selected prey size}

During a first series of experiments, we left all of the insects immobilized on the wall or the white sheet during the day following the night-time use of the light trap. This permitted us to record when $P$. rejecta foragers began to hunt and what size classes of insects they select compared to those available. This study was conducted during three non-consecutive days for three different new moons between 6:00 and 9:30. The comparison of the distribution of the size classes of insects attracted by the light traps and those captured by $P$. rejecta foragers was conducted using the Mann-Whitney test.

\subsection{Predatory behavior}

To study the wasps' predatory behavior, a full repertoire of behavioral acts was first established from observations conducted during preliminary experiments. Referring to this complete list, we recorded each behavioral act performed during predatory sequences. We studied the predatory behavior of $P$. rejecta foragers confronted with three size classes of prey (i.e., 4 mm-long Drosophila, 5-6 mm-long Noctuidae and 9-11 mm-long Noctuidae). To select these prey, between 6:00 and 6:10, as the sun began to rise, we touched each insect of an inappropriate species or size that was immobile on the wall or the white sheet (UV-lit the previous night) with the tip of a fine paintbrush. This process caused them to fly away, so that only appropriate items remained; then, we waited for the arrival of the P. rejecta foragers. 
For each class of prey, we then built a flow diagram where the transition frequencies between behavioral acts were calculated based on the overall number of transitions between each individual behavioral act, a method based on Bayesian procedures (see Dejean, 1980; Bernard et al., 1985).

Using a microscale (Mettler ${ }^{\circledR}$ AE 260$)$, we weighed 25 P. rejecta individuals $(\approx 15 \mathrm{~mm}$ total length) and one of each type of prey tested to calculate the prey-predator weight ratio and verify the upper limit in the selection of insects as prey in terms of size and weight.

During other series of observations of the predatory behavior, we recorded the handling time (from the seizure of a prey until taking off with the prey) corresponding to the capture of one, two and four 4-mm long Drosophila as well as 5-6 and 9-11-mm long noctuids $(\mathrm{N}=18$, $18,15,40$ and 20, respectively). We conducted a log-normal linear model of handling time as a function of prey classes. Model fit was evaluated graphically by exploring residuals patterns (Bolker et al., 2009). A set of t-tests was performed for pairwise comparisons among prey class effects on log-transformed handling time data, and afterwards p-values were adjusted using False Discovery Rate (FDR) criteria. Also, to verify if visual cues alone can be used to detect prey, we made 30 decoys (triangular pieces of grey carton with a base of $4 \mathrm{~mm}$ and height of $10 \mathrm{~mm}$ ) attached to the wall amid the prey using modelling clay.

We also noted during eight days for two new moon periods (involving two different nests), the number of cases when the $P$. rejecta foragers captured and retrieved only one or several (i.e., up to six items) 4-mm long Drosophila. As with the study on the predatory behavior, these small prey were captured on the UV-lit white sheet the previous night whereas, in addition, we placed $\approx 100$ freeze-killed Drosophila on the ground at the base of the sheet. Using R software (R Development Core Team, 2016), we compared the reactions of the first individuals that came to forage on the white sheet (between 6:30 and 7:30) with those that came to forage between $8: 25$ and $8: 35$ and then between 9:25 and 9:35). We compared 
the number of cases when foragers retrieved one, two and up to six small prey using a repeated measure ANOVA and the Tukey post-hoc test (assumptions of normality and homoscedasticity passed).

\subsection{Testing the possibility of nestmate recruitment}

First, during four days (from two days before the new moon to the day following it) and for three non-consecutive new moons (i.e., 12 cases), between 6:30 and 11:30, we registered every hour the number of wasps foraging on the wall that was UV-lit the previous night. Using the R packages car (Fox and Weisberg, 2011) and glmmADMB (Skaug et al., 2014) software, we analyzed the results with a generalized linear mixed model (GLMM) where the response variable (i.e., the number of foraging wasps recorded per time unit) was modeled using discrete hourly time units as fixed factor (five levels) and case identity as random factor. A Poisson error distribution was selected. Model fit was evaluated graphically by exploring residuals patterns. Multiple comparisons between time units were conducted using the Tukey HSD test (R package multcomp; Hothorn et al., 2008).

Second, we tested if a kind of recruitment (e.g., nest-based social facilitation, local enhancement in the field, or both) occurs by noting the number of wasps foraging on the wall at 15:00 after all of the prey had already been captured and again 20 minutes later. The following day, we conducted the same type of verifications but this time we also placed a plate containing 50 freeze-killed Drosophila at the base of the wall at 15:00. This protocol was repeated twice during seven new moon periods plus once for another new moon; 15 cases in total. Using R software, we analyzed the difference between the numbers of wasps foraging once flies were provided, versus non-provided flies situations (considered as a control), by means of a GLM with a Poisson error distribution. Model fit was evaluated graphically by exploring residual patterns. 
In a third series of experiments conducted three times (covering three new moon periods), we aimed to distinguish social facilitation from local enhancement in the field. During "controls" occurring two days before the new moon, we noted the number of wasps foraging on the white sheet (it was UV-lit the previous night) every 5 minutes during 35 minutes from the arrival of the first foraging wasp (around 6:30). The next day (preceding the new moon), each wasp coming to forage between 6:30 and 7:05 was captured and temporarily placed in a tube. This permitted us to record the total number of wasps visiting the sheet every 5 minutes, constituting an experimental lot ("all wasps captured") eliminating both possible local enhancement and social facilitation or any other form of communication. These wasps were freed at the end of the experiment, permitting them to return to their nest; 15 of them were individually marked on the thorax with spots of quick-dry paint (Edding $750 ®$ paint marker) of different colors (see LaPierre et al., 2007). The next night (that of the new moon), the white sheet was not lit to limit the effect of the wasp remembering the presence of this favorable patch (24-hour recall is known in another social wasp species; Moreyra et al., 2016). The day after the new moon, the unmarked $P$. rejecta wasps that came to forage on the white sheet were captured until the first marked one arrived and, during the 20 subsequent minutes, all other wasps were captured (as previously, all of these captured wasps were freed at the end of the experiment). The marked wasp was left free to forage, capture a prey and return to its nest. After 20 minutes, we noted the number of wasps that came to forage every 5 minutes during 35 minutes. So, for this second experimental lot ("first marked wasp free, all others captured") only local enhancement, if any, was eliminated at least at the beginning of the experiment, while possible social facilitation or other kinds of recruitment were permitted. Using $\mathrm{R}$ software, we modeled, with a GLM, the number of foraging wasps from the arrival of the first individual as a function of the covariate length of time (in minutes) combined with the experimental protocol applied factor (fixed factor with three levels: "control", "all wasps 
captured", "first marked wasp free, all others captured"). A Poisson error distribution was selected and a model fit was evaluated graphically by exploring residual patterns. We tested the interaction to detect changes in the temporal pattern of recruitment according to the different experimental protocols. We contrasted model predictions with the least-square means procedure with a fixed significance level of $\alpha=0.05$ using the $R$ package lsmeans software (Lenth, 2016).

Because we again lit the sheet during the next new moon period, we verified if the wasps marked with paint still foraged.

\section{Results and Discussion}

\subsection{Size range of the prey / Cafeteria experiments}

The first $P$. rejecta foragers began to hunt at 6:30 both during preliminary observations and when we noted the range of size of the prey selected by these foragers.

While the length of the insects attracted varied from $2 \mathrm{~mm}$ to $88 \mathrm{~mm}$, the $P$. rejecta foragers captured only 4-14-mm long individuals (Fig. 2). Observations in the field permitted us to note that these wasps mostly hunt small caterpillars, grasshoppers and flies (see also Bichara Filho et al., 2010 for Polybia sericea). So, P. rejecta foragers are able to adapt very quickly to the possibility of a favorable patch, although the insects here were adults (see also the frequent capture of swarming ants and termites by different epiponine species; Richards, 1978; Raveret Richter, 2000; Jeanne and Taylor, 2009; Dejean et al., 2011).

\subsection{Prey capture behavior}

Each tested $P$. rejecta forager likely detected the potential presence of prey from a distance as they flew toward the wall or the plastic sheet covered with insects. When 
approaching the wall, the foraging wasps began with a concentrated search, hovering up and down $\approx 10 \mathrm{~cm}$ from the wall. The contrast of the prey against the wall likely serves as a visual cue because all the 30 decoys made of grey paper were visited by the foraging wasps once or several times (53 visits noted), although they were abandoned immediately after antennal contact.

All types of tested prey can be detected from a distance (up to $8 \mathrm{~cm}$ ) by hovering wasps, but 4-mm long flies and 5-6-mm long noctuids were rather detected by contact after the wasps landed and walked along a sinuous path; the prey, particularly large ones, can be detected from 1-3 cm (Fig. 3). In all cases, detection was followed by a short antennal contact which preceded the seizure of the prey (i.e. classically, the wasps closed their mandibles on the prey body). Then, a stinging phase followed by a backward movement and prey manipulation occurred if the prey did not escape by flying away. The tested prey were taken to the nest at different percentages. However, $1.66 \%$ of the $4-\mathrm{mm}$ long flies and $6 \%$ of the $5-6-\mathrm{mm}$ long noctuids were consumed on the spot before the wasps resumed their sinuous path to capture another or several other item(s). Alternatively, the wasps resumed their sinuous path to capture other items keeping the first ones captured between their mandibles. Larger 9-11-mm long noctuids were never eaten on the spot. Instead, the wasps cut off their wings in $66.7 \%$ of the cases or cut them into two pieces $(16.67 \%)$ and transported one piece immediately. They then returned to collect the other piece (Fig. 3).

Compared to other studies, stinging, or at least flexing the gaster in a movement normally linked to stinging, occurred in all cases (see also Olson, 2000 and papers cited therein), a major difference from most other studies dealing with social wasp predatory behavior (Edwards, 1980; Raveret Richter, 2000). Although the sting-insertion itself was not observable, we deduced that the venom acted when the tip of the wasps' gaster came into contact with the prey because the latter almost immediately stopped struggling. The use of the 
stinger during predation by $P$. rejecta is in contradiction with the fact that sting apparatus autotomy is thought to occur in this wasp species (Overal et al,. 1981). However, we were frequently stung by these wasps and never noted this fact. Moreover, the stingers observed on 50 individuals from eight colonies were smooth (i.e., not barbed), allowing them to sting several times.

As with several other epiponine wasps, $P$. rejecta foragers do not malaxate their prey (Raveret Richter, 2000; Raveret Richter and Jeanne, 1991; Jeanne and Taylor, 2009). When the prey were able to escape, the wasps, seemingly excited, increased both their speed and the sinuosity of their path and immediately seized and stung any newly encountered items and then flew away to take them to their nest (Fig. 3). This behavior increases the probability of finding the escaped prey again and securing it quickly (for non-winged insects). Here, because the targeted prey escaped by flying away, the wasps were able to capture another item with the peculiarity of such excited wasps transporting these heavy prey whole in all cases (while in the "normal situation" the wasps more frequently cut off the wings of these prey or cut them into two pieces; Fig. 3).

\subsection{Load size and prey capture behavior}

Because only $6.66 \%$ of the $9-11-\mathrm{mm}$ long noctuids were transported whole, they likely constituted the maximum load for $P$. rejecta foragers corresponding to $12.60 \pm 0.05 \mathrm{mg}$ (mean $\pm \mathrm{SE} ; \mathrm{N}=25$ items weighed for each type of tested prey and for the wasps). Indeed, the wasps cut off the wings of $66.67 \%$ of other items or cut into two pieces the remaining $16.67 \%$

(Fig. 3) and all larger 12-14-mm long moths captured during the cafeteria experiment (see Fig. 2) were cut into two pieces.

Some $P$. rejecta foragers tended to attain this maximum load by retrieving two 5-6-mm long noctuids $6.24 \pm 0.04 \mathrm{mg}$ each, so $\approx 12.48 \mathrm{mg}$, or up to six 4-mm long flies $2.1 \pm 0.04 \mathrm{mg}$ 
each, so $\approx 12.60 \mathrm{mg}$. Yet, this occurred only for $1.66 \%$ and $2 \%$ of the cases for 4 -mm long flies and 5-6-mm long noctuids, respectively. This maximum weight of prey captured, $\approx 12.60$ $\mathrm{mg}$, corresponds to $30.7 \%$ of the mean $P$. rejecta worker wet weight, or $41.05 \pm 1.5 \mathrm{mg}$. The median size of captured prey being $6 \mathrm{~mm}$ (see Fig. 2 ), thus $\approx 7.00 \mathrm{mg}$, corresponds to $\approx 17.05$ $\%$ of the mean P. rejecta worker wet weight, a value lower than the $24.7 \%$ of the mean prey weight for Polybia sericea (Bichara Filho et al., 2010).

These results were obtained from wasps belonging to a nest situated $105 \mathrm{~m}$ away, but the same studies conducted with foragers from a nest situated $60.5 \mathrm{~m}$ away from the light trap led to in similar results (i.e., up to six small flies retrieved and same maximum load of $\approx 12.60$ mg; very similar percentages as in Fig. 3). However, based on the central place foraging theory, we expected larger loads due to smaller distances. It seems that in the range of their foraging area the $P$. rejecta foragers react similarly to each prey size and weight, at least in the particularly favorable situation of our experimental set up. By comparison, the P. rejecta flight range ( $105 \mathrm{~m} \times 2=210 \mathrm{~m}$ in this study) is high compared to other Polybia species: $P$. scutellaris $150 \mathrm{~m}$; P. occidentalis $126 \mathrm{~m} ;$ P. sericea $75 \mathrm{~m}$ and in the range of that found for two Polistes species (Bichara Filho et al., 2010 and papers cited therein).

The handling time was significantly higher when the prey was heavier (Fig. 4A) and it increased exponentially with the weight of the prey (Fig. 4B). The capture by $P$. rejecta foragers of only one small prey (i.e., 4-mm long Drosophila) rather than several as they can retrieve up to six items (Fig. 3) needs to be considered at the colony level if a form of recruitment is deemed possible. Because the handling time for small prey (i.e., 4-mm long flies) varied significantly with the number of individuals captured (Fig. 4A), we cannot exclude that a given forager favors the recruitment of nestmates and captures only one small prey, while others retrieve several. This might be the case because the individuals that foraged on the white sheet between $6: 30$ and 7:30 retrieved one small prey in $62.5 \%$ of the cases, 
while those that foraged later $(8: 25-8: 35$ and 9:25-9:35) rather retrieved two to six small prey (Fig. 5).

\subsection{Testing if a form of nestmate recruitment exists}

We firstly noted that the number of $P$. rejecta foragers on the wall UV-lit the previous night naturally increased until 9:30 and then decreased as the number of remaining potential prey was extremely reduced (Fig. 6A).

Second, when at 15:00 the wall UV-lit the previous night was devoid of prey, only a few $P$. rejecta continued to forage; this situation remained the same during the 20 subsequent minutes. Yet, when the following day at 15:00 we placed a plate containing 50 freeze-killed Drosophila at the base of the wall, after 20 minutes we noted a significant increase in the number of $P$. rejecta foragers (Fig. 6B). Here, local enhancement is unlikely due to the small number of foragers involved.

Third, during the experiment conducted early in the morning, when the foragers were captured while they hovered in front of the wall, we noted a significantly lower increase in the number of foraging wasps (see "all wasps captured"; social facilitation and local enhancement eliminated; Fig. 6C) in comparison to the two other experimental situations (i.e., "control": all of the wasps were free to forage on the wall, opening up the possibility of multiple recruitments; "first marked wasp free-all others captured": only one wasp marked two days before with paint was allowed to return to its nest where it can eventually recruit nestmates; "all wasps captured": all the wasps were captured as they arrived to forage on the wall, so that they cannot return to their nest and recruit nestmates). Because the number of $P$. rejecta foragers increased the most during the 35-minute monitoring period in the control series (see "Control"; Fig. 6C), a form of recruitment is likely; both social facilitation and local enhancement could be involved in this process. A very similar trend, not significantly 
different, was observed when we left only one marked forager to capture a prey and transport it to the nest (see "First marked wasp free; all others captured"; Fig. 6C); this time, only social facilitation was permitted. This paint-marked forager was found again among nestmates registered first 20-25 minutes later (i.e., 20 minutes corresponds to the first observation recorded in this case), but we cannot assert that tandem flights occurred as suggested for $V$. germanica (Lozada et al., 2016). Indeed, the exponential shape of the cumulative curves in the two last cases is likely the result of a kind of recruitment if compared to the linear shape of the first case.

Therefore, we argue that a kind of recruitment occurred. In this situation, the indication of the general direction from the nest is insufficient due to the presence of buildings between the wasp nests and the targeted favorable patch represented by the wall or the white sheet covered with potential prey. Indeed, one to three flight angles were necessary to go around the buildings and reach the targeted food source. Because local enhancement was eliminated at least during the beginning of the experiment where the first marked wasp remained free and all of the others were captured, social facilitation was involved as is known for other vespid wasps (Hrncir et al., 2007; Schueller and Jeanne, 2012; Taylor et al., 2010, 2012). This is likely facilitated by the ability of foragers to detect food at a relatively long distance (see Raveret Richter, 2000).

Note that during the new moons following these experiments, we never recorded paintmarked $P$. rejecta foragers. This is likely because wasps are able to tear off the paint marks or to the fact that foragers are already old with a short remaining lifespan (only 20-25 days for Polybia occidentalis; O’Donnell and Jeanne, 1992).

In conclusion, $P$. rejecta foragers exhibit a flexible foraging behavior, enabling them to capture several types of prey from favorable patches that were depleted in approximately 3 
hours (06:30-09:30) in our experiments. Experimental evidence supports nest-based social facilitation as a mechanism of recruitment to prey- rich resources.

\section{Acknowledgements}

We are grateful to Andrea Yockey-Dejean for proofreading the manuscript. Financial support for this study was provided by an "Investissement d'Avenir" grants managed by the Agence Nationale de la Recherche (CEBA, ref. ANR-10-LABX-25-01). The authors declare that they have no conflict of interest.

\section{References}

Bernard, J.-M., Blancheteau, M., Rouanet, H., 1985: The predatory behaviour of an earwig, Euborellia moesta (Géné). II: sequential analysis by means of Bayesian inferential methods. Biol. Behav. 10, $1-22$.

Bichara Filho, C.C., Santos, G.M.M., Santos Filho, A.B., Santana-Reis, V.P., Cruz, J.D. da Cruz, Gobbi, N., 2010: Foraging behavior of the swarm-founding wasp Polybia (Trichothorax) sericea (Hymenoptera, Vespidae): daily resource collection activity and flight capacity. Sociobiology 55, 899-907.

Bolker, B. M., Brooks, M. E., Clark, C. J., Geange, S. W., Poulsen, J. R., Stevens, M. H. H., White, J.S. S., 2009: Generalized linear mixed models: a practical guide for ecology and evolution. TREE 24, 127-135.

Brown, R., Payne, A., Graham, K.K., Starks, P.T., 2012: Prey capture and caste-specific payload capacities in the European paper wasp Polistes dominulus. Insectes Soc. 59, 519-525.

Collett, T.S., 1995: Making learning easy: the acquisition of visual information during the orientation flights of social wasps. J. Compar. Physiol. 177, 737-747.

Collett, T.S., Phillipides, A., Hempel de Ibarra, N., 2016: Insect navigation: how do wasps get home? Curr. Biol. 26, R166-168. 
Corbara. B., Carpenter, J.M., Céréghino, R., Leponce, M., Gibernau, M., Dejean, A., 2009: Diversity and nest site selection of social wasps along Guianese forest edges: assessing the influence of arboreal ants. C. R. Biol. 332, 470-479.

Couto, A., Monceau, K., Bonnard, O., Thiéry, D., Sandoz, J.-C., 2014: Olfactory attraction of the hornet Vespa velutina to honeybee colony odors and pheromones. PLoS ONE 9, e115943.

Darracq, A.K., Conner, L.M., Brown, J.S., McCleery, R.A., 2016: Cotton rats alter foraging in response to an invasive ant. PLoS ONE 11, e0163220.

Dejean, A., 1980: Le comportement de prédation de Serrastruma serrula Santschi (Formicidae, Myrmicinae). 2. Etude séquentielle. Ann. Sci. Nat. 2, 145-150.

Dejean, A., Carpenter, J.M., Corbara, B., Wright, P., Roux, 0., LaPierre, L.M., 2012: The hunter becomes the hunted: when cleptobiotic insects are captured by their target ants. Naturwissenschaften 99, 265-273.

Dejean, A., Carpenter, J.M., Gibernau, M., Leponce, M., Corbara, B., 2010: Nest relocation and high mortality rate in a Neotropical social wasp: impact of an exceptionally rainy La Niña year. C. R. Biol. 333, 35-40.

Dejean, A., Céréghino, R., Carpenter, J.M., Corbara, B., Hérault, B., Rossi, V., Leponce, M., Orivel, J., Bonal D., 2011: Climate change impact on Neotropical social wasps. PLoS ONE, 6, e27004.

Donovan, B.J., 2003: Potential manageable exploitation of social wasps, Vespula spp. (Hymenoptera: Vespidae), as generalist predators of insect pests. Internat. J. Pest Manag. 49, 281-285.

Edwards, R., 1980: Social wasps: their biology and control. Rentokil LTD, Felcourt.

Francescato, E., Turillazzi, S., Dejean, A., 1993: Swarming behaviour in Polybioides tabida (Hymenoptera - Vespidae). Actes Coll. Insectes Soc. 8, 121-126.

Fox, J., Weisberg, S., 2011: An R companion to applied regression, $2^{\text {nd }}$ edition. Thousand Oaks, Sage. Gomes, L., Gomes, G., Oliveira, H.G., Morlin Jr, J.J., Desuó, I.C., da Silva, I.M., Shima, S.N., Von Zuben, C.J., 2007: Foraging by Polybia (Trichothorax) ignobilis (Hymenoptera, Vespidae) on flies at animal carcasses. Rev. Bras. Entomol. 51, 389-393. 
Hendrichs, J., Katsoyannos, B.I., Wornoayporn, V., Hendrichs, M.A., 1994: Odour mediated foraging by yellowjacket wasps (Hymenoptera: Vespidae): predation on leks of pheromone-calling Mediterranean fruit fly males (Diptera: Tephritidae). Oecologia 99, 88-94.

Hölldobler, B., Wilson, E.O., 1994: Journey to the ants: a story of scientific exploration. Harvard University Press, Cambridge.

Hrncir, M., Mateus, S., Nascimento, F.S., 2007: Exploitation of carbohydrate food sources in Polybia occidentalis: social cues influence foraging decisions in swarm-founding wasps. Behav. Ecol. Sociobiol. 61, 975-983.

Herre, E.A., Windsor, D.M., Foster, R.B., 1986: Nesting associations of wasps and ants on lowland Peruvian ant-plants. Psyche 93, 321-330.

Howard, K.J., Smith, A.R., O’Donnell, S., Jeanne, R.L., 2002: Novel method of swarm emigration by the epiponine wasp, Apoica pallens (Hymenoptera: Vespidae). Ethol. Ecol. Evol. 14, 365-371.

Hunt, J.H., Jeanne, R.L., Baker, I., Grogan, D.E., 1987: Nutrient dynamics of a swarm-founding social wasp species, Polybia occidentalis (Hymenoptera: Vespidae). Ethology 75,291-305.

Jander, R., 1997: Macroevolution of a fixed action pattern for learning, in Greenberg, G., Tobach, E. (Eds.), The exploration flights of bees and wasps. Garland Publishing, New York, pp 79-100.

Jeanne, R.L., 1972: Social biology of the neotropical Mischocyttarus drewseni. Bull. Mus. Compar. Zool. 144, 63-150.

Jeanne, R.L., 1981: Chemical communication during swarm emigration in the social wasp Polybia sericea (Olivier). Anim. Behav. 29,102-13.

Jeanne, R.L., 1991: The swarm-founding Polistinae. in Ross, K.G., Matthews, R.W. (eds.), Cornell University Press, Ithaca, pp. 191-231.

Jeanne, R.L., Taylor, B.J., 2009: Individual and social foraging in social wasps. in Jarau, S., Hrncir, M. (Eds.). CRC Press, Boca Raton, pp. 53-79.

Kasuya, E., 1982: Central place water collection in a Japanese paper wasp, Polistes chinensis antennalis. Anim. Behav. 30, 1010-1014.

Kramer, D.L., 2001: Foraging behavior, in: Fox, C.W., Roff, D.A., Fairbairn, D.J. (Eds.), Evolutionary ecology, concepts and case studies. Oxford University Press, Oxford, pp 232-246. 
LaPierre, L.M., Hespenheide, H., Dejean, A., 2007: Wasps robbing food from ants: a frequent behavior? Naturwissenschaften 94. 997-1001.

Le Guen, R., Corbara, B., Rossi, V., Azémar, F., Dejean A., 2015: Reciprocal protection from natural enemies in an ant-wasp association. C. R. Biol. 338, 255-259.

Lenth, R.V., 2016: Least-squares means: The R Package lsmeans. J. Stat. Softw. 69, 1-33.

Lozada, M., D’Adamo, P., Buteler, M., Kuperman, M.N., 2016: Social learning in Vespula germanica wasps: do they use collective foraging strategies? PLoS ONE 11, e0152080.

MacArthur, R.H., Pianka, E.R., 1966: On optimal use of a patchy environment. Amer. Nat. 100, 603609.

Moreyra, S., D'Adamo, P., Lozada, M., 2016: Long-term spatial memory in Vespula germanica social wasps: the influence of past experience on foraging behavior. Insect Sci. doi:10.1111/17447917.12366

O'Donnell, S., Hunt, J.H., 2013: Group hunting by workers of two Neotropical swarm-founding paper wasps, Parachartergus apicalis and Agelaia sp. Insectes Soc. 60, 369-372.

O'Donnell, S., Jeanne, R.L., 1992: The effects of colony characteristics on life span and foraging behaviour of individual wasps (Polybia occidentalis, Hymenoptera: Vespidae). Insectes Soc. 39, $73-80$.

Olson, E., 2000: Parachartergus fraternus (Gribodo) (Hymenoptera: Vespidae: Polistinae) uses venom when taking caterpillar prey. Psyche 103, 85-93.

Orians, G.H., Pearson, N.E., 1979: On the theory of central place foraging, in: Horn, D.J., Mitchell, R.D., Stairs, G.R. (Eds.), Analyses of ecological systems. Ohio State University Press, Columbus, pp 154-177.

Overal, W.L., Simoes, D., Gobbi, N., 1981: Colony defense and sting autotomy in Polybia rejecta (Hymenoptera: Vespidae). Rev. Bras. Entomol. 25, 41-47.

Pyke, G.H., Pulliam, H.R., Charnov, E.L., 1977: Optimal foraging: a selective review of theory and tests. Quart. Rev. Biol 52, 137-154. 
Polidori, C., Crottini, A., Della Venezia, L., Selfa, J., Saino, N., Rubolini, D., 2013: Food load manipulation ability shapes flight morphology in females of central-place foraging Hymenoptera. Front. Zool. 10, 36.

R Core Team, 2016: $\mathrm{R}$ a language and environment for statistical computing. $\mathrm{R}$ Foundation for Statistical Computing, Vienna, Austria. URL https://www.R-project.org/

Raveret Richter, M.A., 1990: Hunting social wasp interactions: influence of prey size, arrival order, and wasp species. Ecology 71, 1018-1030.

Raveret Richter, M.A., 2000: Social Wasp (Hymenoptera: Vespidae) foraging: life history, resource distribution, and behavior. Ann. Rev. Entomol. 45, 121-150.

Raveret Richter, M.A., Jeanne R.L., 1985: Predatory behavior of Polybia sericea (Olivier), a tropical social wasp (Hymenoptera: Vespidae). Behav. Ecol. Sociobiol. 16, 165-170.

Raveret Richter, M.A., Jeanne R.L., 1991: Hunting behavior, prey capture, and ant avoidance in the tropical social wasp Polybia sericea. Insectes Soc. 38, 139-147.

Raw, A., 1998: The third trophic level of plant defence: neotropical social wasps' use of odours of fresly damaged leaves when hunting. Rev. Bras. Zool. 15, 1075-1092.

Richards, O.W., 1978: The social wasps of the Americas, excluding the Vespinae. British Museum (Natural History), London.

Santana Jr, P.A., Rocha Gonring, A.H., Picanço, M.C., Soares Ramos, R., Martins, J.C., de Oliveira Ferreira, D., 2012: Natural biological control of Diaphania spp. (Lepidoptera: Crambidae) by social wasps. Sociobiology $59,561-571$.

Santoro, D., Hartley, S., Suckling, D.M., Lester, .PJ., 2015: Nest-based information transfer and foraging activation in the common wasp (Vespula vulgaris). Insectes Soc. 62, 207-217.

Schoener, T.W., 1979: Generality of the size \pm distance relation in models of optimal foraging. Amer. Nat. 114, 902-914.

Schueller, T.I., Jeanne, R.L., 2012: Cue-mediated recruitment in a swarm-founding wasp: successful foragers induce nestmates to search off nest for a scented carbohydrate resource. Psyche 2012, ID 585014. 
Schueller, T.I., Nordheim, E.V., Taylor, B.J., Jeanne, R.L., 2010: The cues have it; nest-based, cuemediated recruitment to carbohydrate resources in a swarm-founding social wasp. Naturwissenschaften 97, 1017-1022.

Shettleworth, S.J., 2010: Cognition, evolution, and behavior. Social learning. Oxford University Press, New York, pp 466-507.

Skaug, H., Fournier, D., Bolker, B., Magnusson, A., Nielsen, A., 2014: Generalized Linear Mixed Models using AD Model Builder. R package version 0.8.0.

Somavilla, A., Fernandes, I.O., Oliveira, M.L., Silveira, O.T., 2013: Association among wasps'colonies, ants and birds in Central Amazonian. Biota Neotrop. 13, 308-313.

Starr, C.K., 1985: A simple pain scale for field comparison of Hymenopterans stings. J. Entomol. Sci. $20,225-232$.

Stephens, D.W., Krebs, J.R., 1986: Foraging theory. Princeton Univ. Press, Princeton.

Stürzl, W., Zeil, J., Boeddeker, N., Hemmi, J.M., 2016: How wasps acquire and use views for homing. Curr. Biol. 26, 470-482.

Sutton, G.J., Hoskins, A.J., Arnould, J.P.Y., 2015: Benefits of group foraging depend on prey type in a small marine predator, the little penguin. PLoS ONE 10, e0144297.

Taylor, B.J., Brus, E.J., Jeanne, R.L., 2012: Introduction of a scented carbohydrate resource into the nest increases departure rate in Polybia occidentalis. Insectes Soc. 59, 151-157.

Taylor, B.J., Schalk, D.R., Jeanne, R.L., 2010: Yellowjackets use nest-based cues to differentially exploit higher-quality resources. Naturwissenschaften 97, 1041-1046.

Wetterer, J.K., 1989: Central place foraging theory: when load size affects travel time. Theor. Pop. Biol. 36, 267-280.

Zeil, J., Kelber, A., Voss, R., 1996: Structure and function of learning flights in bees and wasps. J. Exp. Biol. 199, 245-252. 
Figure legends

Fig. 1. A Polybia rejecta forager marked with a spot of paint.

Fig. 2. Comparison of the percentages of size classes of insects attracted by the light trap and those selected as prey by Polybia rejecta foragers. Statistical comparison conducted from the baseline data; Mann-Whitney test; $\mathrm{N}=45$ in both cases; $\mathrm{U}=390.5 ; \mathrm{P}<0.0001$.

Fig. 3. Flow diagrams representing the sequences of acts during prey capture by the Polybia rejecta foragers. The percentages were calculated from the total number of cases.

Fig. 4. A. Handling time in five situations: flies are 4-mm long Drosophila, small moths are 5-6-mm long noctuids and large moths are 9-11-mm long noctuids. Statistical comparisons: ANOVA $(\mathrm{F}=31.44, \mathrm{P}<0.0001)$; T-test pairwise comparisons post-hoc test adjusted by False Discovery Rate (FDR), different letters indicate a significant difference at $\mathrm{P}<0.01$. B. Relationships between the handling time and the corresponding wet weight of the prey.

Fig. 5. Comparison of the percentages of cases when the Polybia rejecta foragers confronted with small prey (i.e., 4-mm long Drosophila) captured and retrieved one item or several (up to six). Statistical comparisons: ANOVA $(\mathrm{F}=20.25, \mathrm{P}<0.0003)$; Tukey post-hoc test, different letters indicate a significant difference at $\mathrm{P}<0.01$.

Fig. 6. Search for the existence of a kind of recruitment in Polybia rejecta. A. Sequence of the $P$. rejecta forager arriving on the wall lit the night before (means $\pm \mathrm{SE} ; 12$ cases; three nonconsecutive new moons; three replicates per new moon). Statistical comparison: GLM with a Poisson error distribution $(\mathrm{F}=19.78, \mathrm{P}<0.0001)$; Tukey HSD post-hoc test, different letters 
indicate significant differences at $\mathrm{P}<0.05$. B. At 15:00 after the wall was depleted of prey by foraging wasps, residual foraging individuals were counted and again 20 minutes later. The same experiment was conducted the following day, but 50 freeze-killed drosophila were placed at the base of the wall at 14:00. Statistical comparison: GLM with a Poisson error distribution $($ Chi-square $=99.28, \mathrm{P}<0.0001)$. C. Observed counts of foraging wasps and model projections under the three experimental protocols considered (i.e."control", "all wasp captured" and "First marked wasp free; all others captured") arriving between 6:35 and 7:40 on the wall which was lit the previous night. Statistical comparisons: GLM with a Poisson error distribution; Covariate Length of time (Chi-square=190.37, P $<0.0001)$; Factor Experimental protocol $(C h i$-square $=50.48, \mathrm{P}<0.0001)$; Interaction term Length of time $\mathrm{x}$ Experimental protocol (Chi-square $=14.41, \mathrm{P}=0.0007)$. Model prediction slopes contrast by least-square means; Control vs. First marked slopes did not significantly differ. All captured vs. Control and All captured vs. First marked differed at significant level of $\alpha=0$. 


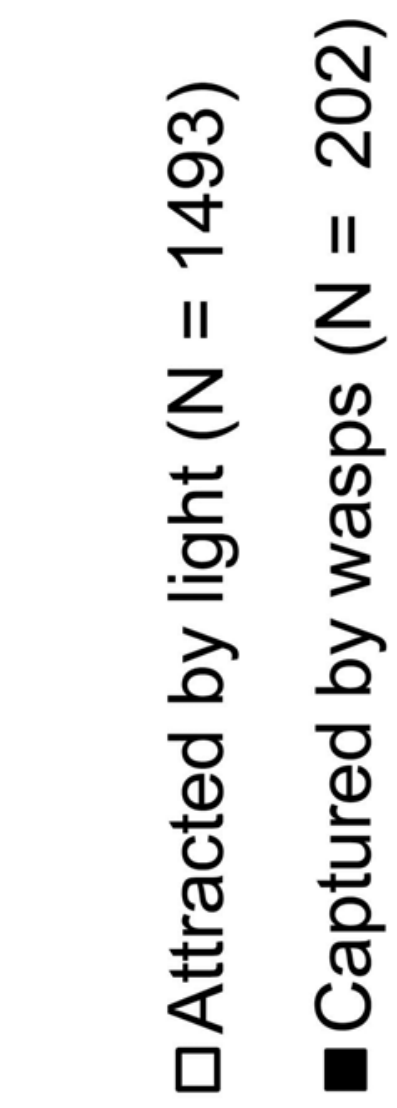

$\infty \varepsilon$

-

으

$\infty$ \%

U

ष (1)

$\sim c$

$\infty{ }^{4}$

0

물

N

(1)

0

10

ใ

రั) 

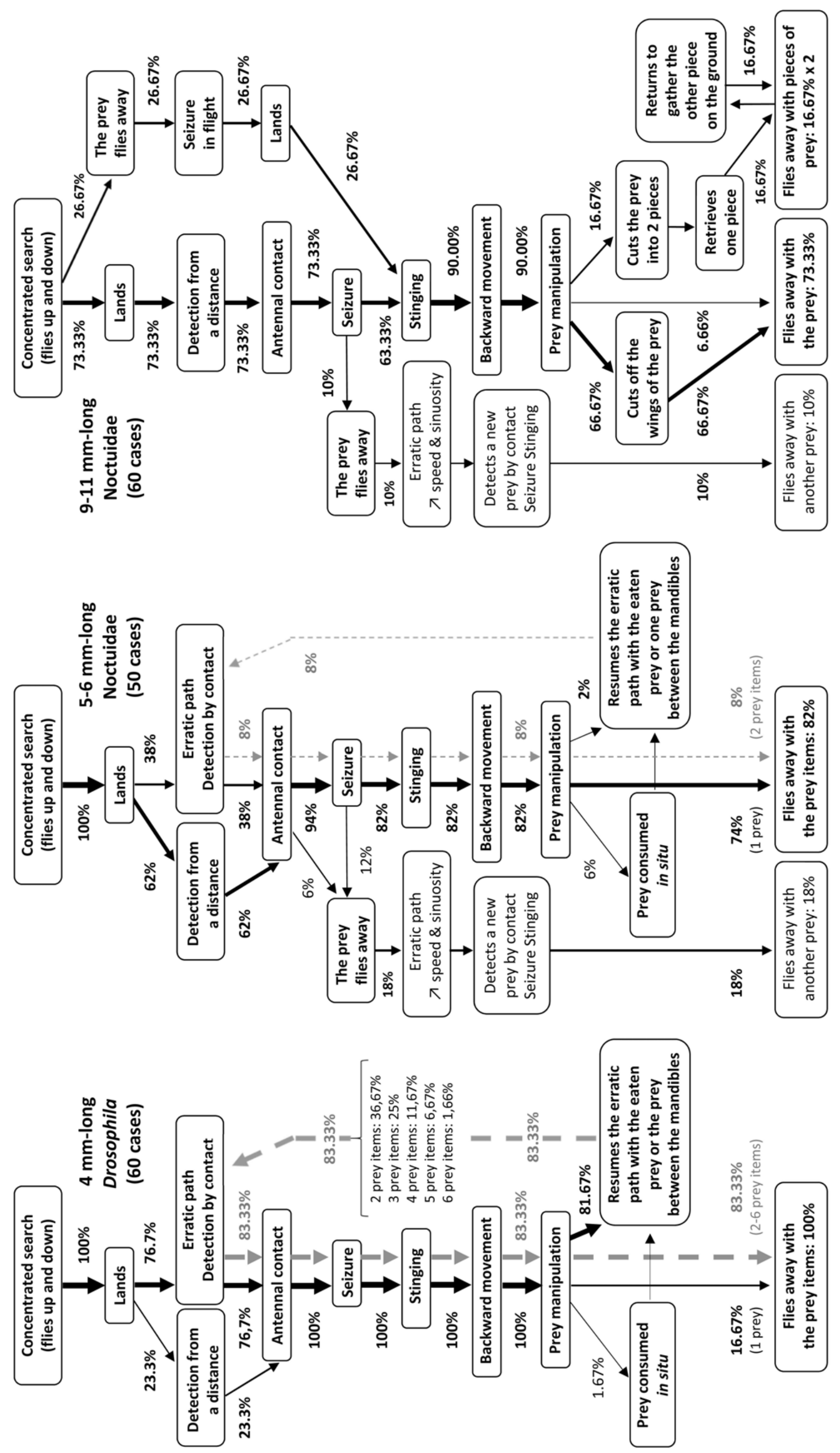

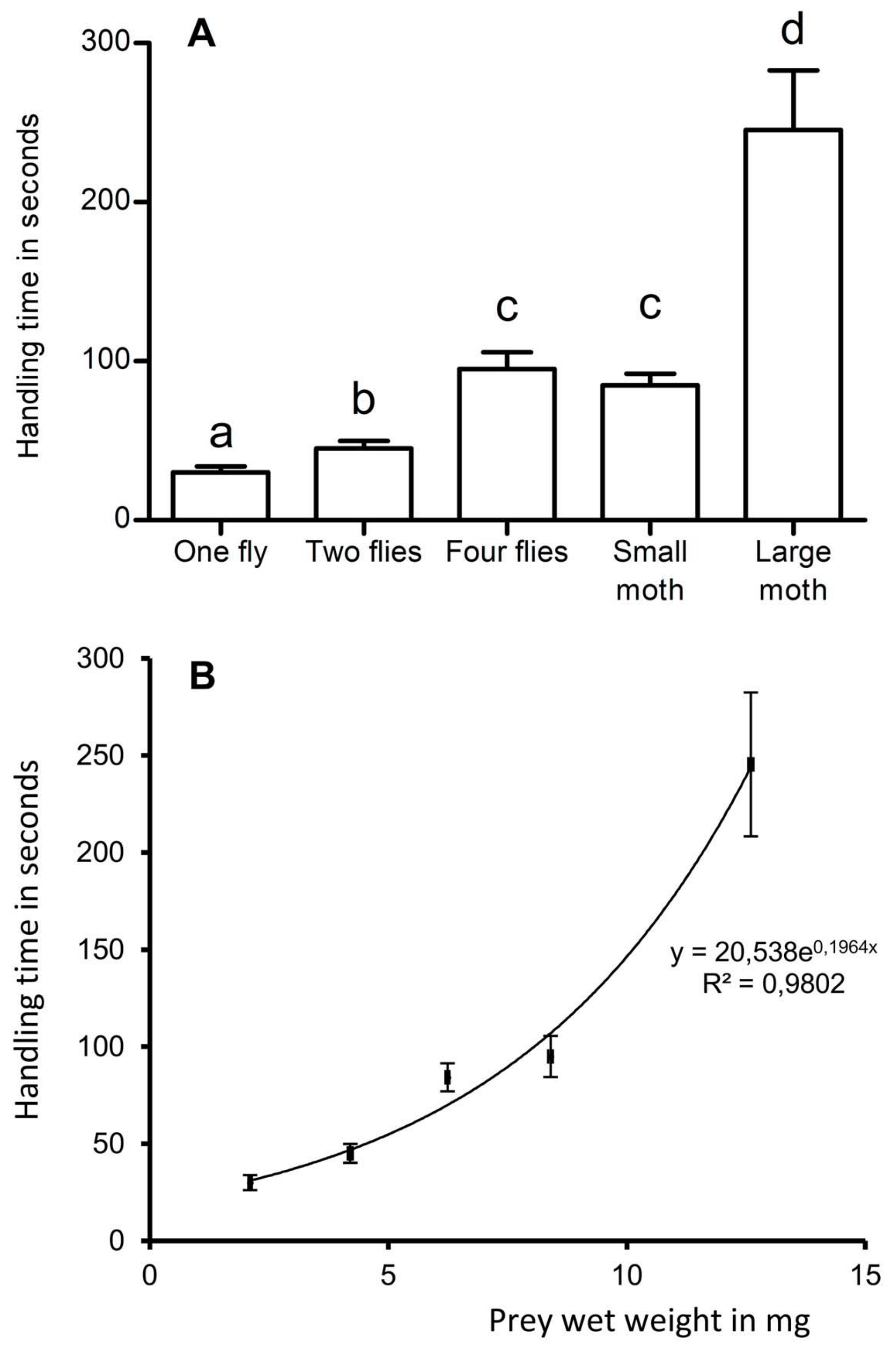
む)

$\infty$

6

II

ঝ

बे

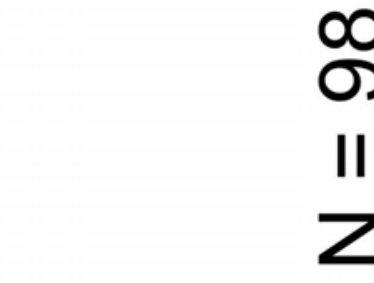

口

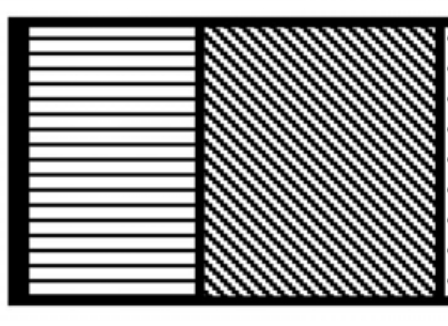

เ

ค?

D)

เด

Q

$\Delta$

$\infty$

$\frac{1}{0}$

$\nabla$

N

0

II

z

$\frac{1}{0}$

ค

$\therefore$

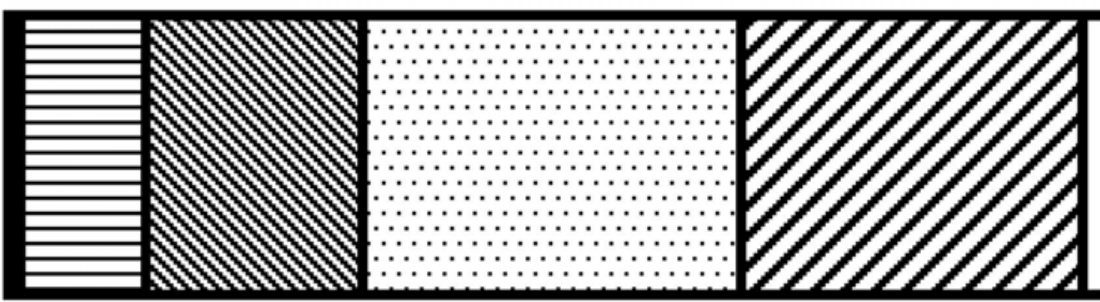

$\frac{1}{0}$
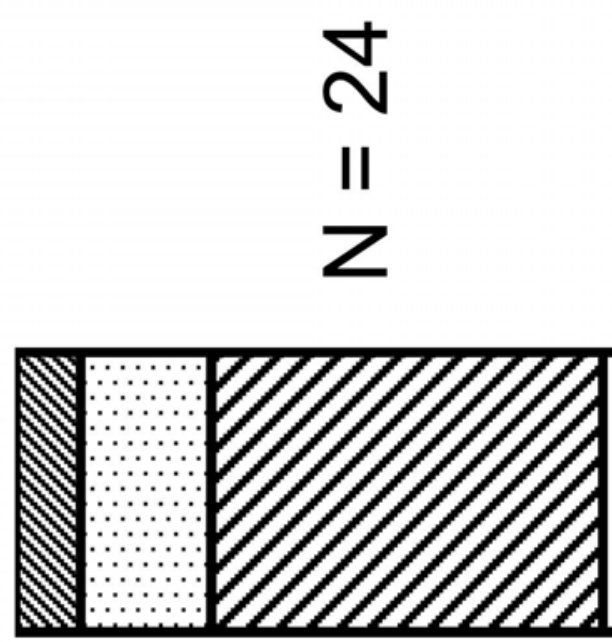

$\frac{1}{6}$

D

문

ஓ̊ํㅇ

○े

8̊ํำ

$0^{0}$

$0^{\circ}$

เ

?

$\infty$

مَ

$\infty$

के

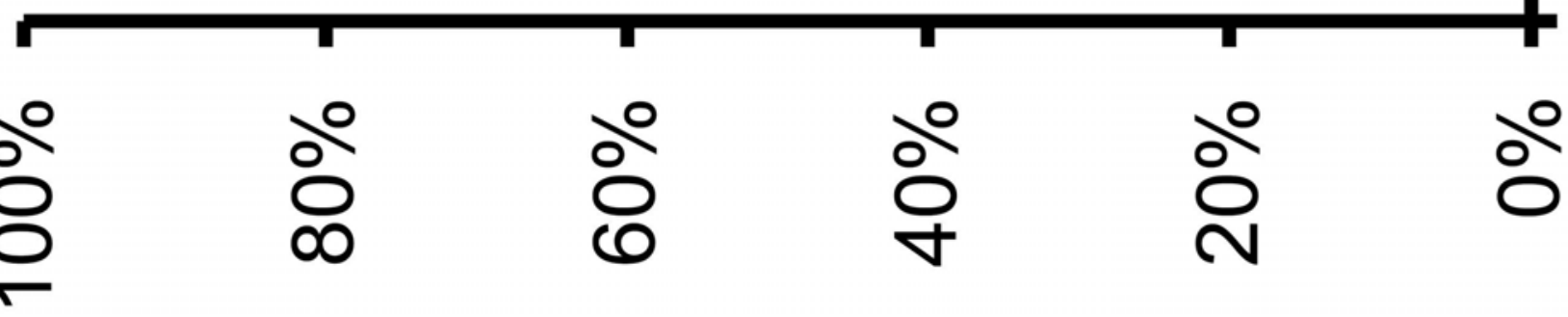



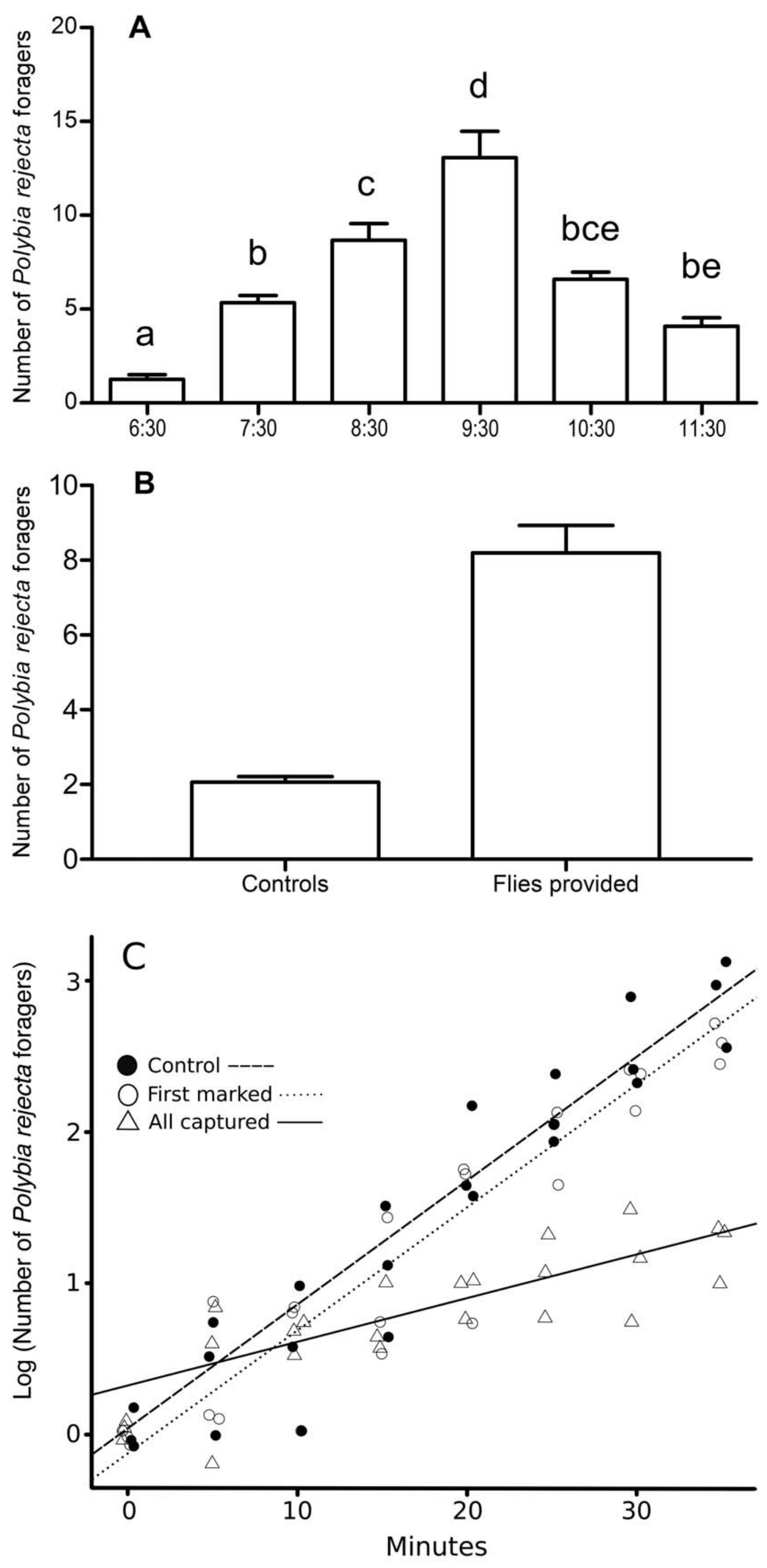